$l_{1}=0, \ldots, l_{h-1}=0, l_{h}=n_{h}$ besitzen. Ein Beispiel liefert hierfür der von

Besicovitch behandelte Fall $m_{k}=a_{k} p_{k}(k=1, \ldots, h)$, wobei

$h \leqslant g, \quad s_{v w}=0 \quad(v, w=1, \ldots, h ; v \neq w), \quad s_{v v}=1 \quad(v=1, \ldots, h)$

wird und (9) mit den $k$ Kongruenzen

$$
l_{v} \equiv 0\left(\bmod n_{v}\right) \quad(v=1, \ldots, k)
$$

gleichbedeutend ist.

Wenn allgemeiner $\mathfrak{R}$ ein beliebiger reeller algebraischer Zahlkörper endlichen Grades ist, so läßt sich die Bedingung (9) ohne weiteres übertragen, indem man die Frauptideale $\left(m_{k}\right)$ in Primideale zorlegt. Für die Bestimmung von $j_{k}$ treten jedoch weitere Bedingungen hinzu, die von der Gruppe der Ideallklassen und der Einheitengruppe herrühren. Deshalb ergibt sich auf diesem Wege doch kein einfaches Verfahren zur Berechnung von $N$, wenn nicht gerade $\Re$ der rationale Zahlkörper ist.

Zum Abschluss sei noch darauf hingewiesen, daß die Voraussetzung der Realität der. Wurzeln beim Beweise nur an einer Stelle benutzt wurde und dort durch die schwächere Annahme ersetzt werden könnte, daß der Körper $\Re_{h}$ keine von 1 und -1 verschiedene Einheitswurzel enthält. Diese Annahme ist zugleich notwendig, wie bereits das einfachste Beispiel

$$
h=1, \quad m_{1}=-4, \quad n_{1}=4, \quad \varrho_{1}=\sqrt[4]{-4}= \pm 1 \pm i, \quad N=4 \neq 2
$$

zeigt.

\title{
Literaturverzeichnis
}

[1] A. S. Besicovitch, On the linear independence of fractional powers of integers, J. London Math. Soc. I5 (1940), S. 3-6.

[2] G. Hajos, Uber einfache und mehrfache Bedectcung des n-dimensionalen Thaumes mit einëm Würfelgitter, Math. Zeitschr. 47 (1941), S. 427-467.

\section{On some exponential sums related to Kloosterman sums}

by

L. J. MORDELI

In memory of Professor Wactaw Sierpinszi

Write

$$
S_{1}(a, b)=S_{1}=\sum_{x=1}^{p-1} e(a x+b \vec{x}), \quad T_{1}=\sum_{x=1}^{p-1} e(a x+b \bar{x})\left(\frac{x}{p}\right),
$$

$$
a b \neq 0(\bmod p),
$$

where $p$ is an odd prime number, $e(x)=e(2 \pi i x / p)$, and we define $\bar{x}$ by $x \bar{x} \equiv 1(\bmod p)$ and often write $\bar{x} \equiv 1 / x(\bmod p)$, and $\left(\frac{x}{p}\right)$ is the Legendre symbol. It is well known that the Kloosterman sum $S_{1}$ satisfies the inequality

$$
\left|S_{1}\right|<2 \sqrt{p},
$$

and that no elementary proof is known $\left({ }^{1}\right)$. However, $T_{1}$ can be evaluated - by elementary methods and

$$
T_{1}=0 \quad \text { if } \quad\left(\frac{a b}{p}\right)=-1,
$$

$$
T_{1}=2 \varepsilon^{-1}\left(\frac{-a}{p}\right) \sqrt{p} \cos (2 \pi h / p) \quad \text { if } \quad\left(\frac{a b}{p}\right)=1,
$$

where $\varepsilon=i^{\left(\frac{p-1}{2}\right)^{2}}$ and $h$ is one solution of

$$
h^{2}-4 a b \equiv 0(\bmod p) \text {. }
$$

This result was first found by Salié [1] in 1931, and another proof has just been given by K. S. Williams [2]. Though their proofs are simple,

(1) Note added in proof by A. Schinzel: An elementary proof has been in the meantime given by S. A. Stiepanov, Trudy Mat. Inst. Stieklov, 112, pp. 346-371. 
the essential ideas used can be expressed in an even simpler way which also leads to other results. Salie's result is included in the

THEOREM 1. Write $T=\sum e(x)$ taken over the solutions of $x^{2} \equiv 4 a b$ $(\bmod p)$. Then if $\left(\frac{a b}{p}\right)=-1, T_{1}=T=0 ;$ and if $\left(\frac{a b}{p}\right)=1$, then

$$
T_{1}=\varepsilon^{-1} \sqrt{p}\left(\frac{-a}{p}\right) T=2 \varepsilon^{-1}\left(\frac{-a}{p}\right) \sqrt{p} \cos (2 \pi h / p)
$$

where $h$ is one root of the congruence $h^{2} \equiv 4 a b(\bmod p)$.

obviously,

$$
p T=\sum_{t, x=0}^{y-1} e\left(x+t\left(x^{2}-4 a b\right)\right)
$$

since the sum in $t$ is zero unless $x^{2}-4 a b \equiv 0(\bmod p)$. The sum in $x$ is a Gaussian sum and so

$$
\sum_{x} e\left(x+t x^{2}\right)=\varepsilon \sqrt{p}\left(\frac{t}{p}\right) e(-1 / 4 t) \text { or } 0 \text { according as } t \neq 0 \text { or } t \equiv 0 \text {. }
$$

Hence $\left({ }^{2}\right)$

$$
p T=\varepsilon \sqrt{p} \sum e(-1 / 4 t-4 a b t)\left(\frac{t}{p}\right)=\varepsilon \sqrt{p}\left(\frac{-b}{p}\right) \sum e(a t+b / t)\left(\frac{t}{p}\right)
$$

on putting $t \rightarrow-t / 4 b$, and so

$$
T_{1}=\varepsilon^{-1}\left(\frac{-b}{p}\right) \sqrt{p} T
$$

This result is easily generalized. We have

THEOREM 2. Put $S=\sum e\left(x_{1}\right)$, where the summation is extended over all the solutions of

$$
x_{1}^{2}+\ldots+x_{n}^{2} \equiv 4 a(\bmod p)
$$

Suppose first that $n$ is odd. Then if $\left(\frac{a}{p}\right)=-1, \$=0$, and if $\left(\frac{a}{p}\right)=1$,

$$
S=2 \varepsilon^{n-1} p^{\frac{n-1}{2}}\left(\frac{-1}{p}\right) \cos (2 \pi h / p)
$$

where $h$ is one solution of $h^{2} \equiv 4 a(\bmod p)$. If $n$ is even,

$$
S=\varepsilon^{n} p^{\frac{n}{2}-1} S_{1}(a, 1)
$$

$\left({ }^{2}\right)$ Here and hereafter we shall omit the limits of summation which will be 0 to $p-1$ for all variables but 0 may be excluded in obvious cases.
We have

$$
p S=\sum_{x, t} e\left(x_{1}+t\left(x_{1}^{2}+\ldots+x_{n}^{2}-4 a\right)\right)
$$

The sum is zero if $t \equiv 0$. If $t \neq 0$, on summing for the $x$, we have

$$
p S=\varepsilon^{n / 2} p^{n / 2} \sum_{t} e(-4 a t-1 / 4 t)\left(\frac{t}{p}\right)^{n} .
$$

The theorem now follows.

Consider next the sums,

$$
\begin{aligned}
S_{2}=\sum_{x, y} e(a x+b y+c \bar{x} y), \quad T_{2}=\sum_{x, y} e(a x+b y+c \bar{x} y) & \left(\frac{x}{p}\right) \\
a b c & \neq 0(\bmod p) .
\end{aligned}
$$

It has been conjectured but not proved that $S_{2}=O(p)$, but only the estimate $S_{2}=O\left(p^{5 / 4}\right)$ is $\mathrm{known}$, and this has been found independently by Hooley, Davenport and Carlitz. I have not seen an estimate for $T_{2}$, but this is easily found from (3), and we have

\section{THEOREM 3 .}

$$
T_{2}=\varepsilon \sqrt{p} \sum_{x} e\left(x+4 a b c / x^{2}\right)=O(p)
$$

For on summing for $x$ and applying equation (3) to (5),

$$
T_{2}=\varepsilon^{-1} \sqrt{p}\left(\frac{-a}{p}\right) \sum_{y, h} e(b y+h),
$$

where $h^{2} \equiv 4 a c \bar{y}(\bmod p)$,

$$
=\varepsilon^{-1} \sqrt{p}\left(\frac{-a}{p}\right) \sum_{h} e\left(h+4 a b c / h^{2}\right)
$$

and this is the result since this exponential sum is $O(p)$.

The sums (5) suggest the consideration of the more general sum

$$
\sum_{3} e\left(a_{1} x_{1}+\ldots+a_{n} x_{n}+a_{n+1} / x_{1} \ldots x_{n}\right)\left(\frac{x_{1} x_{2} \ldots x_{r}}{p}\right), \quad r \leqslant n .
$$

There is no loss of generality in taking $a_{2}=\ldots=a_{n+1}=1$ as is obvious on writing $x_{2} \rightarrow x_{2} / a_{2}$, etc., and so we write

$$
\begin{array}{r}
S_{n}=\sum_{x} e\left(a x_{1}+x_{2}+\ldots+x_{n+1}+1 / x_{1} \ldots x_{n}\right)\left(\frac{x_{1} x_{2} \ldots x_{r}}{p}\right), \\
r \leqslant n, \quad a \neq 0(\bmod p) .
\end{array}
$$

We have now with $\eta=\left(\frac{-a}{p}\right), \zeta=\left(\frac{-1}{p}\right)$, 
TheOREM 4. If $n=2 m, r=m$,

$$
S_{n} /\left(\varepsilon^{-1} \sqrt{p}\right)^{m}=\zeta^{m-1} \eta \sum_{y} e\left(y_{1}+\ldots+y_{m}+4^{m} a / y_{1}^{2} \ldots y_{m}^{2}\right) .
$$

If $n=2 m+1, r=m+1$,

$$
S_{n} /\left(\varepsilon^{-1} \sqrt{p}\right)^{m+1}=0 \quad \text { if } \quad\left(\frac{a}{p}\right)=-1
$$

but if $\left(\frac{a}{p}\right)=1$, and $h^{2}=4 a(\bmod p)$,

$$
S_{n} /\left(\varepsilon^{-1} \sqrt{p}\right)^{m+1}=\zeta^{m} \eta \sum_{y, h} e\left(y_{1}+\ldots+y_{m}+2^{m} h / y_{1} \ldots y_{m}\right) .
$$

Sum (7) for $x_{1}$. Then from (3),

$$
S_{n} / \varepsilon^{-1} \sqrt{p}=\eta \sum e\left(y_{1}+x_{2}+\ldots+x_{n}\right)\left(\frac{x_{2} \ldots x_{r}}{p}\right), \quad y_{1}^{2} \equiv 4 a / x_{2} \ldots x_{n} \text {. }
$$

Substitute for $x_{n}$. Then

$$
S_{n} / \varepsilon^{-1} \sqrt{p}=\eta \sum e\left(y_{1}+x_{2}+\ldots+x_{n-1}+4 a / y_{1}^{2} x_{2} \ldots x_{n-1}\right)\left(\frac{x_{2} \ldots x_{r}}{p}\right) \text {. }
$$

Sum for $x_{2}$. Then

$$
\begin{aligned}
& S_{n} /\left(\varepsilon^{-1} \sqrt{p}\right)^{2}=\zeta \eta \sum e\left(y_{1}+y_{2}+x_{3}+\ldots+x_{n-1}\right)\left(\frac{x_{3} \ldots x_{r}}{p}\right), \\
& y_{2}^{2} \equiv 4^{2} a / y_{1}^{2} x_{3} \ldots x_{n-1}
\end{aligned}
$$

Substitute for $x_{n-1}$. Then

$$
\begin{aligned}
& S_{n} /\left(\varepsilon^{-1} \sqrt{p}\right)^{2} \\
& \quad=\zeta \eta \sum e\left(y_{1}+y_{2}+x_{3}+\ldots+x_{n-2}+4^{2} a / y_{1}^{2} y_{2}^{2} x_{3} \ldots x_{n-2}\right)\left(\frac{x_{3} \ldots x_{r}}{p}\right)
\end{aligned}
$$

We continue the process, summing for $x_{3}$, etc.

If $n=2 m, r=m$, we come to a stage,

$$
S_{n} /\left(\varepsilon^{-1} \sqrt{p}\right)^{n_{2}}=\zeta^{m-1} \eta \sum e\left(y_{1}+\ldots+y_{m}+x_{m+1}\right), \quad y_{m}^{2}=4^{m} a / y_{1}^{2} \ldots y_{m-1}^{2} x_{m+1}
$$

$$
=\zeta^{m-1} \eta \sum e\left(y_{1}+\ldots+y_{m}+4^{m} a / y_{1}^{2} \ldots y_{m}^{2}\right) \text {. }
$$

If $n=2 m+1, r=m+1$, we come to a stage,

$S_{n} /(\varepsilon \sqrt{p})^{m}=\zeta^{m-1} \eta \sum e\left(y_{1}+\ldots+y_{m}+x_{m+1}+4^{m} a / y_{1}^{2} \ldots y_{m}^{2} x_{m+1}\right)\left(\frac{x_{m+1}}{p}\right)$.

Then summing for $x_{m+1}$,

$$
S_{n} /\left(\varepsilon^{-1} \sqrt{p}\right)^{m+1}=\zeta^{m} \eta \sum e\left(y_{1}+\ldots+y_{m}+y_{m+1}\right)
$$

where

$$
y_{m+1}^{2}=4^{m+1} a / y_{1}^{2} \ldots y_{m}^{2},
$$

and so the sum is zero unless $\left(\frac{a}{p}\right)=1$. If $h^{2} \equiv 4 a$,

and so

$$
y_{1} \ldots y_{m+1} \equiv \pm 2^{m} h
$$

$S_{n} /\left(\varepsilon^{-1} \sqrt{p}\right)^{m+1}=\zeta^{m} \eta \sum_{y, h} e\left(y_{1}+\ldots+y_{m}+2^{m} h / y_{1} \ldots y_{m}\right), \quad h^{2} \equiv 4 a(\bmod p)$

In particular, when $n=4$,

$\sum_{x} e\left(a x_{1}+x_{2}+x_{3}+x_{4}+1 / x_{1} x_{2} x_{3} x_{4}\right)\left(\frac{x_{1} x_{2}}{p}\right)=\left(\frac{a}{p}\right) p \sum_{y} e\left(y_{1}+y_{2}+16 a / y_{1}^{2} y_{2}^{2}\right)$

when $n=5$,

$$
\begin{aligned}
\sum_{x} e\left(a x_{1}+x_{2}+x_{3}+x_{4}+x_{5}+1 / x_{1} x_{2} x_{3} x_{4} x_{5}\right)\left(\frac{x_{1} x_{2} x_{3}}{p}\right) \\
=\varepsilon \eta p^{3 / 2} \sum_{y, h} e\left(y_{1}+y_{2}+4 h / y_{1} y_{2}\right) .
\end{aligned}
$$

\section{References}

[1] Hans Salié, Über die Kloostermansehen Summen $S(u, v: q)$, Math. Zeitsehr. 34 (1931), pp. 91-109.

[2] K. S. Williams, Finite transformation formulae involving the Legendre symbol, Pacific Journ. Math. 34 (1970), pp. 559-568.

\section{UNIVERSITY OF OALGARY, Canada}

t. JOHN'S COLLFGE, Cambridge, England 